\title{
A review of the European species of Phradis (Hymenoptera: Ichneumonidae: Tersilochinae), with a description of a new species from Spain
}

\author{
AndRey I. KHALAIM ${ }^{1}$, SANTiago BORDERA ${ }^{2}$ and AleXander RODRÍGUEZ-BERRÍO ${ }^{3}$
}

\begin{abstract}
${ }^{1}$ División de Estudios de Postgrado e Investigación, UAM Agronomía y Ciencias, Universidad Autónoma de Tamaulipas, Cd. Victoria 87149, México; Zoological Institute of the Russian Academy of Sciences, Universitetskaya nab. 1, St. Petersburg 199034, Russia; e-mail: hymenopt@zin.ru

${ }^{2}$ Instituto de Investigación de Biodiversidad CIBIO, Universidad de Alicante, Ap. Corr. 99, 03080-Alicante, Spain; e-mail: s.bordera@ua.es

${ }^{3}$ Departamento Académico de Entomología, Facultad de Agronomía, Universidad Nacional Agraria La Molina, Av. La Molina s/n, Distrito la Molina, Lima 12, Perú; e-mail: arodriber@lamolina.edu.pe
\end{abstract}

Key words. Hymenoptera, Ichneumonidae, Tersilochinae, Phradis, new species, taxonomy, key, Europe, Spain

\begin{abstract}
European species of the genus Phradis Förster, 1869 are reviewed. A new species from Spain, Phradis mediterraneus sp. n., is described and illustrated. A key to all 24 European species and data on distribution and biology of these species are pr ovided.
\end{abstract}

\section{INTRODUCTION}

Phradis Förster is a moderate sized genus comprising 38 Palearctic species (Khalaim, 2007a), including a new species described in this paper, a single described Nearctic species (Khalaim, 2002) and two species from the Afrotropical region (Khalaim, 2007b). Undescribed species are also reported from Australia (Gauld, 1984), China and Vietnam (A.I. Khalaim, unpubl.). Most species occur in the Holarctic region, but Nearctic species are mostly undescribed (Townes, 1971). Twelve European species were revised and keyed by Horstmann (1971). Moreover, eleven new species were described after the revisions by Horstmann (1981), Aubert (1986), Horstmann \& Kolarov (1988) and Khalaim (2004, 2007a). Studying material from a mountainous area on the East Mediterranean coast of Spain, one more species, Phradis mediterraneus sp. n., was found. An original key to all 24 European species is provided. In addition, the data on distribution and biology of the European species is updated.

The genus Phradis gives name to a generic group which also includes the genera Heterocola Förster, 1868 and Palpator Khalaim, 2006. All of them are characterised by the first metasomal segment without glymma, propodeum with basal area, mesosoma without or with weak sternaulus, and forewing with the second recurrent vein usually interstitial or antefurcal (Horstmann, 1971). Within this group, Phradis is easily recognized by the interstitial second recurrent vein and the short maxillary and labial palpi, which are extremely elongate in both of the other two genera. Phradis includes mostly small sized species with body length usually not exceeding 2.0-4.0 $\mathrm{mm}$. Some species of the genus are known as common parasitoids of sap beetle larvae (Nitidulidae: Meligethes spp.) on rape. Flight period of adults is from early spring to the middle of summer, but some species fly until autumn.

\section{MATERIAL AND METHODS}

A large number of Phradis specimens were examined from the collections of the Natural History Museum, London, UK (BMNH), Zoologischen Staatsammlung München, Germany (ZSM), Museo Nacional de Ciencias Naturales, Madrid, Spain (MNCN), Entomological Collection of the University of Alicante, Spain (CEUA), Institute of Zoology, Kiev, Ukraine (SIZK), Institute of Ecology, Vilnius, Lithuania (IEV), Zoological Institute of the Russian Academy of Sciences, St. Petersburg, Russia (ZISP), Zoological Museum of Moscow State University, Moscow, Russia (ZMUM). Some type and non-type specimens were also borrowed from K. Horstmann (Würzburg, Germany; HC), M. Schwarz (Kierchlag, Austria; SC), A. Lozan (Czech Republic; LC), J. Sawoniewicz (Białystok, Poland; SWC) and A.E. Humala (Petrozavodsk, Russia; HMC). To save space, we have omitted the abbreviation for the material from ZISP.

Types of the following species were studied: Phradis corsicator Aubert and Phradis toreador Aubert (Musée Cantonal de Zoologie, Lausanne, Switzerland; MZLS), P. decrescens (Thomson), Thersilochus temporalis Thomson [= Phradis brevis (Brischke)] and P. morionellus (Holmgren) (Museum of Zoology, Lund University, Sweden; MZLU), P. denticulatus Khalaim, $P$. longibasalis Khalaim, $P$. pesenkoi Khalaim and $P$. vinosus Khalaim (ZISP).

Material of $P$. mediterraneus sp. $\mathrm{n}$. was collected by means of Malaise traps in several habitats in the Sierra Mariola Natural Park, Sierra Carrasqueta and Font Roja Natural Park (Spain). The Malaise traps operated from May to September 2001 and from April to October 2002. In all cases, trapped insects were removed fortnightly. A specimen collected in Sierra Carrasqueta by mean of a hand net in 2006, was also included.

Type material of the new species is preserved in CEUA and ZISP.

Scanning electron microscopy studies were done using a Hitachi S-3000N in low vacuum mode. Terms used in descrip- 
tions for surface microsculpture of sclerites were adapted from Eady (1968). Terminology for morphological structures mainly follows Townes (1969), and Gauld \& Bolton (1988) for insect body divisions. The term "basal area" is used for the combined basal area and areola (area superomedia). These areas, if present, are combined in the Tersilochinae. Taxonomy and distribution are as in the catalogue TaxaPad (Yu et al., 2005). Geographic data listed in "Material" are given by countries, arranged in alphabetic order. Place-names are mainly those used in the atlas Microsoft Encarta Premium 2006.

\section{RESULTS}

\section{Genus Phradis Förster, 1869}

Phradis Förster, 1869: 148. Type species: Thersilochus (Phradis) brevis Brischke, 1880.

Eutomus Förster, 1869: 148 (nec Hope, 1838, Carabidae; nec Lacordaire, 1866, Curculionidae). Type species: Isurgus lanceolatus Szépligeti, 1899 (=Thersilochus morionellus Holmgren, 1860).

Isurgus Förster, 1869: 148. Type species: Isurgus lanceolatus Szépligeti, 1899 (=Thersilochus morionellus Holmgren, 1860).

\section{Key to European species of Phradis (females only)}

1 Flagellum of antenna with 19-22 segments. Ovipositor sheath about 1.2 times as long as first tergite. Body length usually $5-6 \mathrm{~mm} \ldots \ldots \ldots \ldots \ldots \ldots \ldots \ldots \ldots \ldots \ldots \ldots \ldots \ldots$

- Flagellum with 8-17 segments. Length of ovipositor sheath variable. Body length usually $2-4 \mathrm{~mm} . \ldots \ldots \ldots \ldots . \ldots 3$

2 Malar space longer than basal width of mandible. Temple almost as long as eye width in dorsal view. Frons and mesopleuron densely granulate, impunctate. Metacarpus not reaching apex of fore wing, ending far from it. Petiole of first tergite compressed, strongly striate laterally. Antenna entirely dark; metasoma dark brown to black. Ovipositor with very thin needle-like tip (Fig. 8) . . . . . . . P. gibbus

- Malar space shorter than basal width of mandible. Temple about 0.6 times as long as width of eye (Fig. 1). Frons densely punctate, granulate; mesosoma distinctly punctate, predominantly smooth and shining. Metacarpus almost reaching apex of fore wing. Petiole of first tergite round in transverse section, entirely smooth. Antenna yellowish basally; metasoma behind first tergite yellow ventrally to dark brown dorsally. Ovipositor with weak dorsal subapical depression, without needle-like tip.......... longibasalis

3 Ovipositor sheath at least 2.4 times as long as first tergite. Ovipositor with small and narrow dorsal subapical notch (Fig. 13), distinctly upcurved. Flagellum of antenna with 9-14 segments, slender (Fig. 2). Mesopleuron granulate, impunctate. Metasoma dark brown to black. ......... 4

- Ovipositor sheath twice as long as first tergite, or shorter. Other characters varied. ................ 5

4 Flagellum with 9-11 segments, basal flagellomeres very slender (Fig. 2). Basal area of propodeum narrow, distinctly elongate..................... minutus

- Flagellum with 13-14 segments, basal flagellomeres moderately slender. Basal area of propodeum wide, about as wide as long. ..................... pesenkoi

5 Second recurrent vein entirely absent (Fig. 36). Basal area of propodeum as long as apical area (Figs 34, 35). Upper tooth of mandible much shorter than lower tooth. Flagellum with 13 segments (Fig. 32). Clypeus entirely smooth. Sternaulus distinct, linear, reaching middle of mesopleuron. . ...

........................... Poreador
Second recurrent vein present, only unpigmented in its anterior part. Basal area of propodeum shorter than apical area. Upper tooth of mandible longer than or subequal to lower tooth. Other characters variable. . . . . . . . . . . . . . 6

6 Flagellum with $8-12$ segments. . . . . . . . . . . . 7

- Flagellum with 13-17 segments. . . . . . . . . . . . . 16

7 Dorsolateral areas of propodeum smooth and shiny. Flagellum with 11 segments (rarely with 12 segments), usually yellowish basally. Propodeum with narrow and short basal area, which is usually indistinct or represented by a furrow. Malar space shorter than 0.3 the basal width of mandible. First metasomal segment entirely smooth. Ovipositor sheath about $1.6-1.8$ times as long as first tergite. . . . . . . . 8

- Dorsolateral areas of propodeum granulate. Other characters variable......................... 9

8 Basal flagellomeres slender, 4th flagellomere twice as long as wide. Ovipositor with roundish dorsal subapical notch. . . Basal flagellomeres stouter (Fig. 3), 4th flagellomere shorter. Ovipositor with narrow dorsal subapical notch (Fig. 10)........................ nigritulus

9 Flagellum with 8-10 segments, basal flagellomeres very slender (Fig. 4). Antenna yellow basally. Ovipositor sheath almost twice as long as first tergite. Metasoma behind first segment yellow to dark brown, never black. Second recurrent vein usually slightly antefurcal. .......P. decameron

- Flagellum with 11-12 segments. Antenna entirely black or ovipositor sheath shorter. Metasoma sometimes entirely black. Second recurrent vein variable. . . . . . . . . . 10

10 Ovipositor tip distinctly denticulate dorsally (Fig. 11). Basal area of propodeum indistinct, about as long as wide, often substituted by rugulose furrow. . . . . . . P. denticulatus

- Ovipositor tip not denticulate dorsally............ 11

11 Mesopleuron finely punctate on a smooth or finely granulate surface. Sternaulus distinct. Ovipositor with a very thin needle-like tip (Fig. 9), its sheath somewhat longer than first tergite. ....................... terebrator - Mesopleuron granulate, impunctate. Sternaulus absent or indistinct. Ovipositor not as above. . . . . . . . . 12

12 Base of antenna slender (Fig. 5); first flagellar segment usually more than 3 times as long as wide........... 13

- Base of antenna stout (Fig. 7); first flagellar segment usually not reaching 2.7 times as long as wide.......... 14

13 All trochanters and hind femur black or strongly darkened. Antenna entirely black. Ovipositor sheath $1.7-1.8$ times as long as first tergite............... monticola

- Trochanters and hind femur not darkened (sometimes hind femur brownish). Antenna yellowish or brownish basally. Ovipositor sheath shorter, about 1.1-1.2 times as long as first tergite. ................ rufiventris

14 Ovipositor sheath short, $0.9-1.1$ the length of first tergite. Flagellum stumpy, 3rd and 4th flagellomeres usually less than 1.5 times as long as wide (Fig. 7). Trochanters, hind femur and antenna black or strongly infuscate..........

P. brevicornis Ovipositor sheath longer, usually $1.3-1.6$ the length of first tergite. Flagellum slender, if stumpy, then trochanters, hind femur and antenna basally more or less yellowish. . . . . 15

15 Flagellum with 12 segments. Trochanters and hind femur not darkened (sometimes hind femur brownish). Antenna yellowish or brownish basally. Ovipositor sheath 1.5-1.6 the length of first tergite. . . . . . . . . . . P. thyridialis - Flagellum with 11 segments. All trochanters and hind femur black or strongly darkened (hind femur much darker than tibia). Antenna entirely black. Ovipositor sheath $1.3-1.4$ the length of first tergite. .............. P. decrescens 


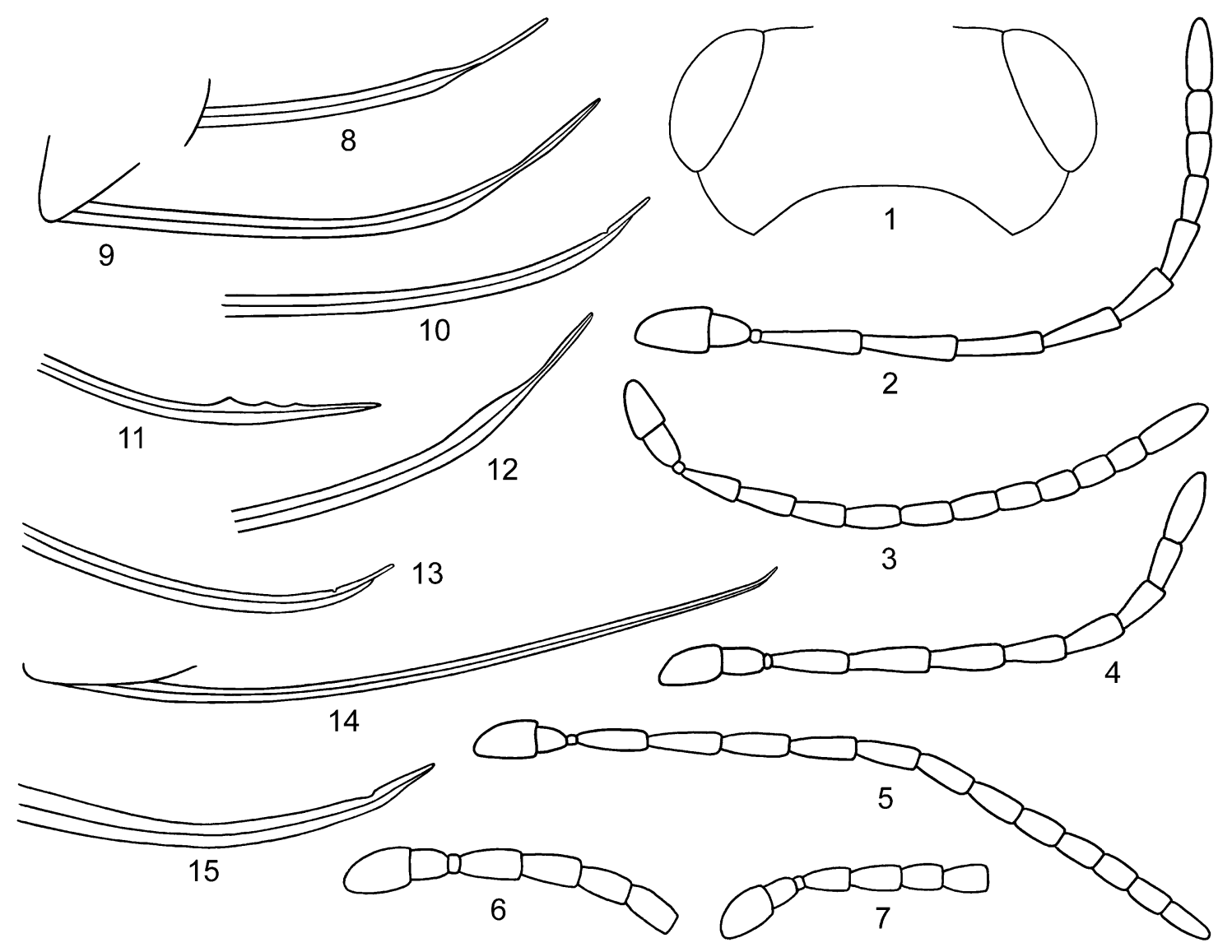

Figs 1-15: Morphology of females of Phradis species. P. longibasalis (1), P. minutus (2, 13), P. nigritulus (3, 10), P. decameron (4), P. monticola (5), P. polonicus (6), P. brevicornis (7), P. gibbus (8), P. terebrator (9), P. denticulatus (11), P. punctus (12), P. interstitialis (14), P. brevis (15). 1 - head, dorsal view; 2-7 - antenna, lateral view; 8-15-apex of ovipositor, lateral view.

16 Ovipositor with long and very thin needle-like tip (Figs 9, 12), its sheath $1.0-1.5$ times as long as first tergite. . . . . 17

- Ovipositor without thin needle-like tip, length of sheath variable........................... 20

17 Legs yellow except for black hind coxa. Antenna with scape and pedicel yellow and flagellum brownish. Dorsolateral areas of propodeum very finely granulate. ...... P. vinosus

- All coxae, trochanters and hind femur (except apex) strongly darkened. Antenna entirely black. Dorsolateral areas of propodeum sometimes irregularly wrinkled.... 18

18 Flagellum with 13 segments. Dorsolateral areas of propodeum finely granulate. . . . . . . . . . . . . . terebrator

- Flagellum with 15-17 segments. Dorsolateral areas of propodeum as a rule irregularly wrinkled. . . . . . . . . . . 19

19 Mesopleuron entirely granulate or sometimes with a smooth central area, finely punctate (sometimes indistinctly). Ovipositor sheath usually not longer than first tergite. . . . . . . . P. punctipleuris

- Mesopleuron smooth, densely and entirely punctate. Ovipositor sheath 1.5 times as long as first tergite... P. punctus

20 Thyridia transverse, rarely as long as wide. Flagellum with 13-15 segments, all flagellomeres distinctly elongate. First tergite more or less depressed, distinctly striate laterally. Metasoma behind first segment dark brown. Ovipositor sheath 1.4-1.8 times as long as first tergite. . P. morionellus

- Thyridia more or less elongate, if about as long as wide, then other characters not as above. . . . . . . . . . . . 21

21 Antenna entirely black, flagellum slender (Figs 5, 22). Ovipositor with dorsal subapical notch (Figs 19, 27). . . . 22
Antenna yellowish basally, if black (sometimes in P. interstitialis), then ovipositor very thin and without dorsal subapical notch (Fig. 14). . . . . . . . . . . . . . . 24

22 Basal area of propodeum transverse, about twice as wide as long. Ovipositor sheath about as long as first tergite (Fig. 26). Flagellum with 13-14 segments (Fig. 22).......... ...................... P. mediterraneus sp. n.

- Basal area of propodeum distinctly elongate, about 1.5 times as long as wide (sometimes indistinct). Ovipositor sheath almost twice as long as first tergite. . . . . . . . . . . 23

23 Flagellum with 13 segments............. P. monticola - Flagellum with $15-16$ segments. ........... P. corsicator

24 Ovipositor thin, evenly narrowing towards a very thin strongly upcurved apex (Fig. 14); sheath 1.4-2.0 times as long as first tergite. Flagellum with 14-16 segments, dark, basal flagellomeres short. .......... P. interstitialis - Ovipositor moderately thick (Fig. 15); sheath at most 1.6 times as long as first tergite. Other characters varied. . . 25

25 Basal area of propodeum long and narrow, about twice as long as wide. Antenna strongly darkened (almost black); flagellum with 15 segments, basal flagellomeres moderately slender (Fig. 6). Ovipositor sheath somewhat shorter than first tergite. . . . . . . . . . . . . . . . Polonicus Basal area of propodeum about as long as wide. Antenna more or less yellowish or pale-brown basally, rarely entirely dark; flagellum with 13-14 segments (very rarely with 15 segments), basal flagellomeres sometimes more slender. Ovipositor sheath 1.0-1.6 times as long as first tergite. . 26 

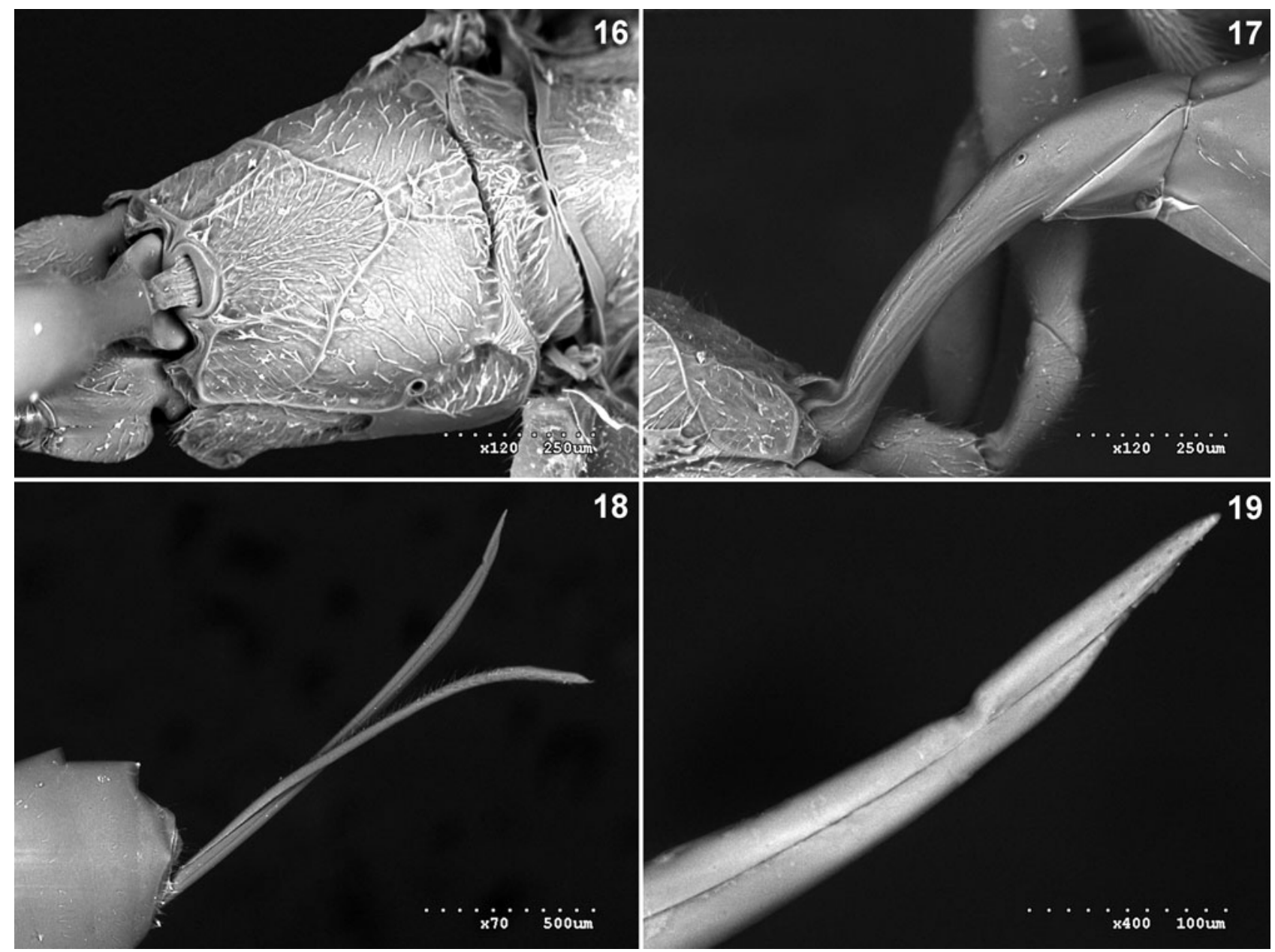

Figs 16-19: Morphology of P. corsicator, female, holotype (SEM photos). 16 - propodeum, dorsal view; 17 - first metasomal segment, lateral view; 18 - ovipositor, lateral view; 19 - apex of ovipositor, lateral view.

26 Ovipositor sheath 1.4-1.6 times as long as first tergite. Temple more or less evenly granulate, without a shiny area. Basal area of propodeum about as long as wide. .

............................. thyridialis

- Ovipositor sheath $1.0-1.3$ times as long as first tergite. Temple finely granulate, partly almost smooth and shiny. Basal area of propodeum more or less elongate, usually about 1.5 times as long as wide. ........... Previs

\section{P. brevicornis Horstmann, 1971}

Phradis brevicornis Horstmann, 1971. Holotype + .

Material. $139 q$ and $\delta$ examined. Austria: Steiermark (Haus, Kemeterhof, $1200 \mathrm{~m}$ [ZSM]). Hungary: Kétvölgy. Italy. Trentino-Alto Adige [Südtirol] (Parcines [Partschins], 700-1050 m, 2\%, paratypes [ZSM]; $35 \mathrm{~km}$ E Trento, Castello Tesino, $1200 \mathrm{~m}, 1$ 으, paratype [ZSM]; $15 \mathrm{~km} \mathrm{~W}$ Rowereto, Riva del Garda, 800-1000 m, 3 0 , paratypes [ZSM]), Veneto (N Vicenza, Piovene, 900-1300 m [ZSM]). East Kazakhstan: Tarbagatay Mts, Staropyatigorskoye. Lithuania [IEV]: Pasvalys; Panevėžys; NE Vilnius, Raudondvaris; Smalvos. Russia: Karelia (N Onega lake, Povenets), Novgorod reg. (20 km NW Pestovo, Tychkino), Smolensk reg. (Smolenskoe Pohozerye national park, Przheval'skoye), Yaroslavl' reg. (25 km S Yaroslavl', "Berditsino"), Krasnodar reg. (NW Sochi, Lazarevskoe), Bashkortostan reg. (Belebey), Chelyabinsk reg. (Il'menskiy nature reserve), Chita reg., Amur reg., Jewish Autonomous Region, Primorskiy reg., Kurile Islands (Kunashir Is., Shikotan Is.),
Magadan reg., Chukotka. Spain: Madrid (El Ventorrillo, $1480 \mathrm{~m}$ [MNCN]). Ukraine: Poltava reg. (Alexandrovka), Crimea (S Simferopol', Chatyrdag Mts), Donetsk reg. (N Slov'yans'k, Bogorodichnoe).

Distribution. Transpalaearctic species. Europe, Kazakhstan, Russian Siberia and Far East.

Biology. Host unknown. Flight period in Europe from May to early August.

\section{P. brevis (Brischke, 1880)}

Thersilochus (Phradis) brevis Brischke, 1880. Type lost.

Thersilochus (Thersilochus) temporalis Thomson, 1889. Lectotype ?.

Thersilochus styriacus Strobl, 1904. Syntypes 2 ô. Lectotype not designated.

Material. $198 \uparrow$ and $\delta \hat{~ e x a m i n e d . ~ A r m e n i a: ~ T s a v . ~ A u s t r i a: ~}$ Niederösterreich [Lower Austria] (SW Reichenau, Semmering pass [BMNH]). East Azerbaijan: Altyagach, 1200-1400 m [SIZK]. Bulgaria [ZSM]: Rhodope Mts (Sadovo; Rila Monastery; Bachkovo; "Tšervena skala"; "Bezovo"; "Er-kjupria"). England: NE Cambridge, Wicken [BMNH]. Georgia: $10 \mathrm{~km} \mathrm{~W}$ Adigeni, Goderdzi pass. Germany: Type of Thersilochus temporalis Thomson [MZLU], Lower Saxony (Göttingen [ZSM]), Bavaria (Würzburg [ZSM]; Nürnberg [ZSM]; Peissenberg [ZSM]; Starnberg, Kerschlach [ZSM]; env. Freising, Erching [ZSM]), Baden-Württemberg (Stuttgart [ZSM]; Bad Herrenalb, Schwarzwald [Black Forest] [ZSM]). Kazakhstan: Eastern- 

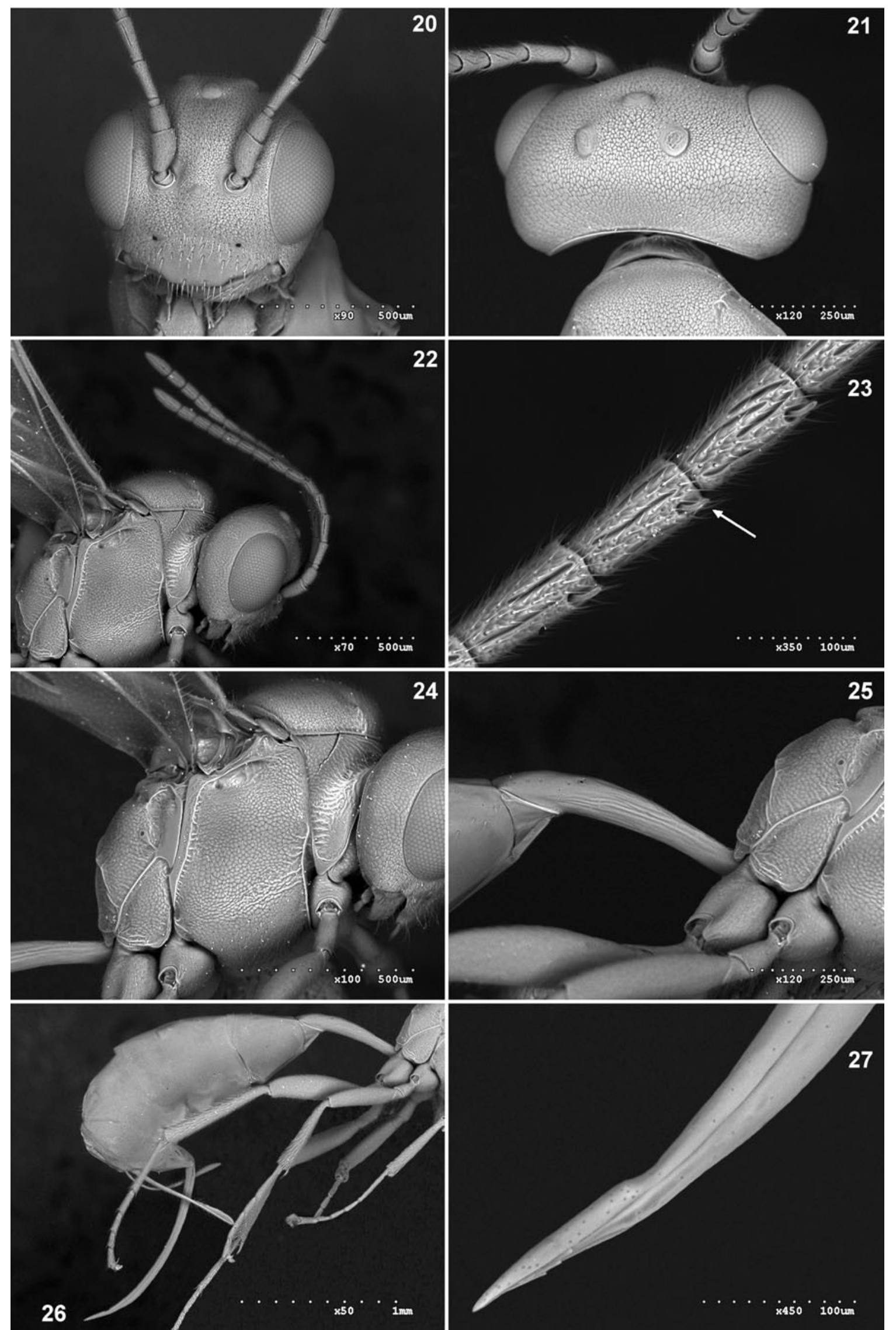

Figs 20-27: Morphology of P. mediterraneus sp. n., female (SEM photos). 20 - head, frontal view; 21 - head, dorsal view; 22 mesosoma and head with antennae, lateral view; 23 - middle flagellar segments; 24 - mesosoma, lateral view; 25 - first metasomal segment, lateral view; 26 - metasoma with ovipositor, lateral view; 27 - apex of ovipositor, lateral view. 
Kazakhstan reg. (18 km SE Zaysan, Saur Temirsu Mts; $10 \mathrm{~km}$ SE Zaisansk, $1800 \mathrm{~m}$ ); Staropyatigorskoe, Tarbagatay Mts, 1200-2200 m. Lithuania [IEV]: Alytus; Žuvintai; near Vilnius, Pilaite. Mongolia. East Poland: Hajnówka [SWC]. Russia: Leningrad reg. (50 km S St. Petersburg, Susanino), Novgorod reg. (20 km NW Pestovo, Tychkino), Krasnodar reg. (Gelendzhik [IEV]; NW Sochi, Lazarevskoe [IEV]; Krasnaya Polyana, 2000 $\mathrm{m}$ [SC, SIZK]), Adygea (Caucasian nature reserve, Guzeripl'), Karachay-Cherkessia (Arkhyz), Stavropol' reg. (Teberda nature reserve), Kabardino-Balkaria (Verkhnyaya Balkariya), Alania [North Ossetia] (Ardon; Vladikavkaz [SIZK]), Voronezh reg. (Borisoglebsk [ZMUM]), Irkutsk reg., Chita reg., Khabarovsk reg., Primorskiy reg., Kurile Islands (Kunashir Is.), Kamchatka reg. Switzerland: "Schwaba" [ZSM]. Ukraine [SIZK]: Ternopil' reg. (Medobory nature reserve), Kiev.

Distribution. Common transpalaearctic species. Present in almost the whole of Europe, Caucasus, Kazakhstan, Mongolia, Russian southern Siberia and Far East.

Biology. Parasitoid of Meligethes difficilis Heer (Horstmann, 1981; Williams et al., 1984). Flight period from April to July.

\section{P. corsicator Aubert, 1969}

Phradis minutus corsicator Aubert, 1969. Holotype 우.

(Figs 16-19)

Material. France: Corsica (Bastia, 1 9 , holotype [MZLS]). Spain: Castilla-La Macha (Cabañeros National Park, 19 [CEUA]).

Distribution. Western Europe (Spain, France).

Biology. Host unknown. Flight in May.

\section{P. decameron Khalaim, 2004}

Phradis decameron Khalaim, 2004. Holotype + , lost.

Material. Armenia: Meghri, 1500-2000 m, 2 ㅇ [SIZK, ZISP]. Russia: Stavropol' reg. (Teberda nature reserve, 1 ㅇ). Ukraine: Sumy reg. (Mikhailovskaya Tselina nature reserve, 29 , holotype and paratype).

Distribution. Eastern Europe (Ukraine) and Caucasus.

Biology. Host unknown. Flight in June and July.

\section{P. decrescens (Thomson, 1889)}

Thersilochus (Thersilochus) decrescens Thomson, 1889. Lectotype + .

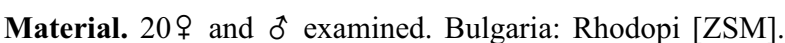
France: "Kofa". Germany: "Germany" (type) [MZLU], Bavaria (Siglohe [ZSM]). Georgia: Borjomi. Kazakhstan: WesternKazakhstan reg. (Mirgorodki), Eastern-Kazakhstan reg. (Buran - Akzhar; 18 km SE Zaysan, Saur Temirsu Mts). Russia: Leningrad reg. (S St. Petersburg, Mozhayskiy).

Distribution. Europe, Caucasus (Georgia), Kazakhstan.

Biology. Flight period from late May to late July.

\section{P. denticulatus Khalaim, 2007}

Phradis deticulatus Khalaim, 2007a. Holotype 9 .

Material. $11 \%$ and $3 \delta^{\hat{\sigma}}$ examined (holotype and paratypes). Kazakhstan: Qaraghandy [Karaganda] reg. (Karkarala Mts [ZISP, SIZK]). Russia: Bashkortostan (Bashkiriya nature reserve [ZISP, SIZK]). Slovenia: Bohinjska Bela [BMNH]. Ukraine: Ternopil' reg. (Medobory nature reserve [ZISP, SIZK]).

Distribution. Eastern Europe, Kazakhstan.
Biology. Host unknown. Flight period from late May to early July.

\section{P. gibbus (Holmgren, 1860)}

Thersilochus gibbus Holmgren, 1860. Holotype ot.

Phradis grandis Hellén, 1958. Holotype 9.

Material. Finland: $30 \mathrm{~km}$ E Hämeenlinna, Lammi, 10 đ [HMC]. Russia: Leningrad reg. (55 km S St. Petersburg, 19 ; NW St. Petersburg, Levashevo, 1 \%), Novgorod reg. (Tigoda, 1 ㅇ), Primorskiy reg. ( 1 o $)$.

Distribution. Transpalaeacrtic species. Europe, Russian Siberia and south of Far East, ?China (Shanxi reg.: Chao, 1976).

Biology. Host unknown. Flight in Europe from mid April to early June.

\section{P. interstitialis (Thomson, 1889)}

Thersilochus (Thersilochus) interstitialis Thomson, 1889. Lectotype $\hat{\delta}$.

Isurgus brachygaster Szépligeti, 1899. Lectotype + .

Material. $58 \uparrow$ and $\hat{\delta}$ examined. Austria: Niederösterreich (SW Reichenau, Semmering pass [BMNH]). Belarus: S Minsk, Loshitsa [ZMUM]. Bulgaria: Rhodope Mts (Vitosha national park [ZSM]). England: S Cambridge, Trumpington, on Brassica juncea infested with Meligethes aeneus \& Ceuthorhynchus sp. [BMNH]. Georgia: $20 \mathrm{~km}$ NE Adigeni; Borjomi. Germany [ZSM]: Lower Saxony (Hedemünden; Wiershausen), Bavaria (Würzburg; Polling; Gauting; Herrsching, Widdersberg; Zorneding), Hessen (Lahntal; Staufenberg). Italy: Lombardy (Brescia, $370 \mathrm{~m}$ ), Basilicata ("Tremosine Vesio, Nalbo", 800-1060 m [ZSM]). Lithuania [IEV]: Ažuoliniai [Bambininkai]; Karkliniškès. Moldova: Kotovsk [Kotovskoe]. Poland [SWC]: Hajnówka; Mielnik; Siemiatycze. Russia: Leningrad reg. (70 km N St. Petersburg, Sosnovo), Alania (Vladikavkaz [SIZK]), Dagestan (Rutul). Spain: Madrid (Sierra de Guadarrama; Villaverde) [MNCN]. Tadzhikistan: Gissarskiy Mts, Kondara canyon. Ukraine: Luhans'k reg. (15 km E Sverdlovs'k, Proval'skaya steppe nature reserve; Antratsit).

Distribution. Europe, Caucasus, Central Asia (Tadzhikistan).

Biology. Parasitoid of Meligethes aeneus F., M. coracinus Sturm, M. difficilis Heer, M. nigrescens Stephens (=picipes Sturm) and M. viridescens F. (Nitidulidae) (Horstmann, 1981; Nilsson \& Andreasson, 1987; Šedivý, 1983; Williams et al., 1984). Flight period in Europe from late April to mid of July.

\section{P. longibasalis Khalaim, 2007}

Phradis longibasalis Khalaim, 2007. Holotype + .

Material. $15 q$ and 10 examined (holotype and paratypes). Poland: "Topiło FD94 at Hajnówka", 1 [SWC]. Russia: Smolensk reg. (Smolenskoe Pohozerye national park, Przheval'skoye, 1 \%), Khabarovsk reg., Primorskiy reg., Kurile Islands (Kunashir Is.).

Distribution. Transpalaearctic species. Rare in Europe (Poland and Smolensk reg. of Russia) and more abundant in the Russian Far East.

Biology. Host unknown. Flight in Europe from May to June.

\section{$P$. mediterraneus sp. $\mathrm{n}$.}

(Figs 20-31)

Diagnosis. This new species resembles $P$. monticola and $P$. corsicator in having entirely black antenna with a 

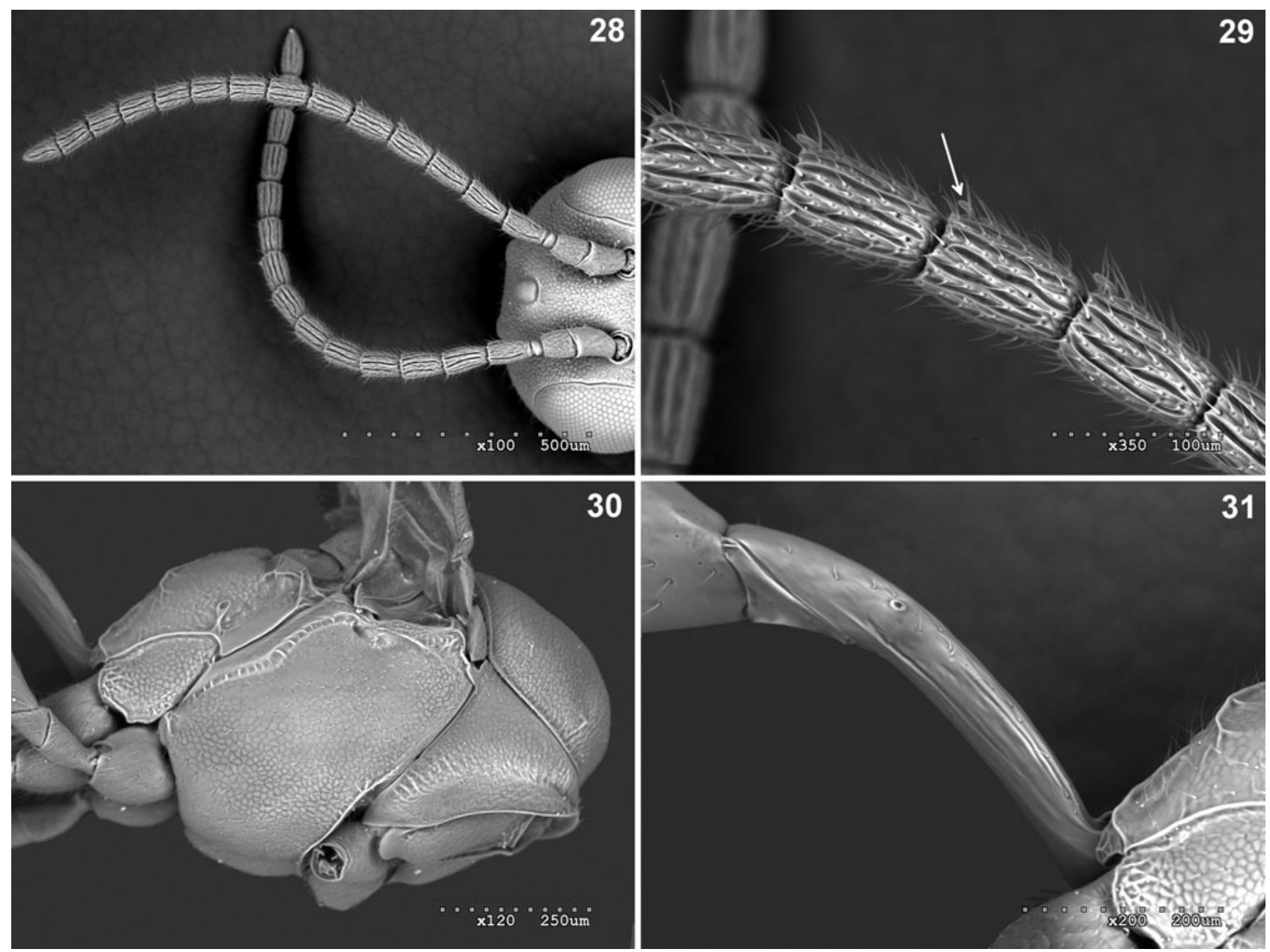

Figs 28-31: Morphology of P. mediterraneus sp. n., male (SEM photos). 28 - antennae, frontal view; 29 - middle flagellar segments; 30 - mesosoma, lateral view; 31 - first metasomal segment, lateral view.

slender flagellum of 13-14 segments (Fig. 22), second recurrent vein entirely present, basal area of propodeum shorter than apical area, thyridia elongate, ovipositor with conspicuous dorsal subapical notch (Fig. 27). It differs from them by the basal area of propodeum, which is transverse, about twice as wide as long, and by its ovipositor sheath which is about as long as first tergite (Fig. 26).

Description. Female (Figs 20-27): Body length (without ovipositor) $3.0-4.0 \mathrm{~mm}$. Forewing $2.0-2.4 \mathrm{~mm}$ long.

Measurement of holotype. Body length $3.7 \mathrm{~mm}$. Forewing $2.5 \mathrm{~mm}$ long. Head $0.4 \mathrm{~mm}$ long and $0.8 \mathrm{~mm}$ wide. Mesosoma $1.1 \mathrm{~mm}$ long, $0.7 \mathrm{~mm}$ wide (mesoscutum). First tergite $0.6 \mathrm{~mm}$ long; postpetiole $0.2 \mathrm{~mm}$ long and $0.1 \mathrm{~mm}$ wide. Second tergite $0.4 \mathrm{~mm}$. Ovipositor sheath $0.7 \mathrm{~mm}$ long.

Head transverse in dorsal view, 1.8-2.0 times as wide as long, slightly constricted behind eyes (Fig. 21). Temple rounded, 0.7-0.9 times as long as width of eye (viewed from above) (Fig. 21). Vertex, frons, temple, face and cheek slightly but densely punctate on a strongly granulate background. Occiput smooth and shiny. Malar space about $0.7-0.8$ times as long as basal width of mandible. Clypeus 2.8-3.1 times as wide as long, slightly convex with thick and dense punctures on a granulate background on the upper half, where long silver setae are present; lower half smooth and shiny, its lower margin straight centrally with a dense line of long and strong silver setae. Mandible very long, with subparallel rims, teeth of equal length and dense setiferous punctures at base, rest smooth and polished. Antennal flagellum with 13-14 segments (Fig. 22), distinctly widened from base to apex; first flagellomere twice as long as wide; fourth to sixth flagellomeres with apical finger-shaped external structures (Fig. 23 ). Hind ocellus separated from eye by a distance about 2.0-2.3 times its diameter. Space between hind ocelli 2.2-2.6 times their diameter.

Mesosoma (Fig. 24) slightly but densely punctate on a strongly granulate background (Fig. 24). Pronotum longitudinally strigose on lower lateral side. Epomia absent. Notaulus weak and short, its length about $0.2-0.3$ the distance to scutellum. Scutellar groove without longitudinal carinae. Scutellum with lateral carinae present along its basal half. Upper division of metapleurum smooth and shiny. Sternaulus indistinct. Propodeal spiracle very small, rounded, separated from pleural carina by 3.0-4.0 times its diameter. Propodeum with a pair of median longitudinal carinae bounding the basal area laterally. Pleural carina, lateral parts of apical transverse carina and dorsal 

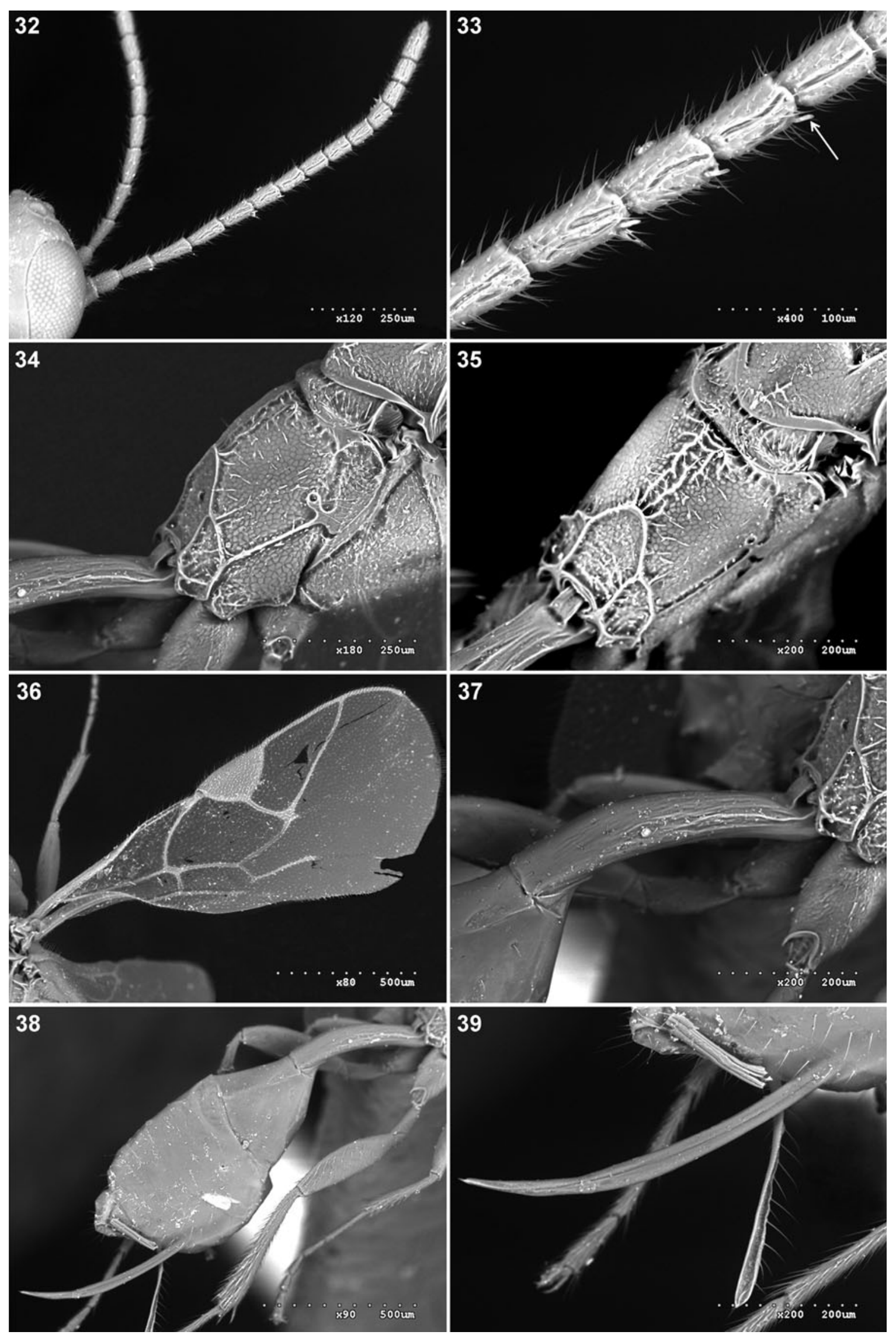

Figs 32-39: Morphology of P. toreador, female, holotype (SEM photos). 32 - antennae, lateral view; 33 - middle flagellar segments; 34 - propodeum, dorso-lateral view; 35 - propodeum, dorsal view; 36 - forewing; 37 - first metasomal segment, dorsolateral view; 38 - metasoma with ovipositor, dorso-lateral view; 39 - apex of ovipositor, lateral view. 
part of basal transverse carina conspicuously defined. Basal area rugose, small and transverse, 2.0-2.2 times as wide as long, its length $0.2-0.3$ the length of apical area.

Wings. Ramulus absent. Metacarpus long and strongly sclerotized, reaching 0.8 the distance to apex of fore wing. Pterostigma very big, triangular and strongly sclerotized. Second recurrent vein interstitial, weakly inclivous and with one bulla. Nervulus opposite basal vein. Nervellus reclivous, not intercepted by discoidella, which is absent. Mediella unpigmented, indistinct in basal half. Brachiella absent.

Legs slender. Tarsal claws not pectinate. Hind femur 3.6-3.7 times as long as broad.

Metasoma strongly compressed. First tergite smooth and shiny dorsally with few sparse setiferous punctures laterally. Petiole sometimes with long lateral longitudinal striae (Fig. 25); longitudinal carinae indistinct. Glymma absent. Postpetiole 1.5-1.7 times as long as its maximum width (measured dorsally), spiracle placed clearly behind middle. Second to last metasomal segments smooth and shiny. Thyridia long and narrow, 3.5-4.0 times as long as its maximum basal width. Ovipositor upcurved, with distinct dorsal subapical notch and two or three inconspicuous small teeth at apex of ventral valve (Fig. 27). Ovipositor sheath as long as hind tibia and about the same length as first tergite (Fig. 26). Ovipositor tip short, thin and very sharp, 2.5-2.8 times as long as depth of middle section of ovipositor.

Coloration. Body black. Mandible, palpi, tegula, legs except coxae and trochanters, metasoma behind first segment and sometimes lower half of clypeus red-brown. Pterostigma and sometimes hind femur, and basal part of front and middle femora dark brown. Wings hyaline.

Male (Figs 28-31): Body length 2.2-2.5 mm. Forewing 1.2-1.8 mm long. Head transverse, 1.8-2.1 times as wide as long; temple 0.7-0.9 times as long as eye. Malar space about 0.6-0.7 times as long as basal width of mandible. Clypeus 2.9-3.0 times as wide as long, with long silver setae at base, central part of apical margin straight and with a dense line of long and strong silver setae as in female. Antennal flagellum with 14 segments (Fig. 28); first flagellomere 1.8-2.3 times as long as wide, fourth to sixth flagellomeres with apical external finger like structures as in female. Tyloids absent. Hind ocellus separated from eye by about twice its diameter. Space between hind ocelli about twice their diameter. Other head features and mesosoma microsculpture as in female. Hind femur 3.7-3.8 times as long as broad. Metasoma strongly compressed, tergites smooth and shiny. First tergite with few sparse setiferous punctures laterally. Petiole sometimes with long longitudinal striae laterally (Fig. 31). Postpetiole 1.5 times as long as its maximum width. Thyridia elliptic, 1.7-2.0 as long as its maximum width. Body coloration as in female.

Habitat. This species was found in Caveta del Buitre (Sierra Mariola, U.T.M coordinates: 30SYH161937), an area located $1200 \mathrm{~m}$ a.s.l. with a vegetation dominated by Pinus halepensis Miller, isolated trees of Quercus ilex L. subsp. rotundifolia (Lam.) Schwartz ex T. Morais and shrubs of Quercus coccifera L., all in herbaceous pasture dominated by Brachypodium retusum Pers. In Sierra de Carrasqueta the species was found in habitats in Mas de San Ignacio (940 m, U.T.M.: 30SYH187766) and Venta Carrasqueta (980 m, U.T.M.: 30SYH191770), in both cases, the cultivated areas were abandoned about ten and twenty years ago respectively. In the former the dominant vegetation is brushwood belonging to the TeucrioUlicetum dianii Mesomediterranean association, and in the latter, the dominant vegetation is a residual Mesomediterranean holm-oak wood of Quercetum rotundifoliae association with Quercus rotundifolia Lam., Rubia peregrina L. and Smilax aspera L. and an herbaceous stratum of Brachypodium retusum Pers.

In Font-Roja Natural Park, the species was collected on the Menejador Peak (1352 m, U.T.M.: 30SYH143819). In this habitat, the dominant vegetation belongs to the association Salvio-Erinaceetum anthyllidis.

The climate in all these mountains is Mediterranean, characterised by a dry summer, annual mean temperatures between $14-16^{\circ} \mathrm{C}$, with absolute minimum temperatures in winter of $-12^{\circ} \mathrm{C}$ and maximum in summer of $40-44^{\circ} \mathrm{C}$. Rainfall is about $500-700 \mathrm{~mm}$ per year with maximums in spring and autumn.

Type material. Holotype: $q$, Sierra de Mariola (Alicante, Spain), Caveta de Buitre, 23.v.2001, $1200 \mathrm{~m}$, leg. CIBIO [CEUA]. Paratypes: Sierra de Mariola: Caveta de Buitre (1200 m.s.n.m), 23.v.2000, $2 q$, leg. CIBIO (1 $q$ in CEUA, 19 in ZISP). Sierra de Carrasqueta-Font Roja (Alicante, Spain): Mas de San Ignacio (940 m.s.n.m), 23.v.2001, 10, leg CIBIO [CEUA], Menejador (1352 m.s.n.m), 23.v.2001, 1 , leg. CIBIO [CEUA], 15.v.2002, 1 \%, leg. CIBIO [ZISP], Malaise trap. Mas de San Ignacio (940 m.s.n.m), 21.v.2006, 10, leg. S. Bordera [CEUA]), hand net.

Distribution. Spain.

Etymology. The species name refers to the Mediterranean Spanish Region, the area where the material was collected.

Biology. Hosts unknown. This species seems to be univoltine, with a maximum flight activity in May.

\section{P. minutus (Bridgman, 1989)}

Thersilochus minutus Bridgman, 1889. Lectotype 우.

Material. $75 q$ and $\delta$ examined. Armenia: Art'ik, $1600 \mathrm{~m}$ [SIZK]. Austria [ZSM]: Tirol (Imsterberg, 1250 m; Kössen, 600 $\mathrm{m}$; "Obsteig", $1000 \mathrm{~m}$ ). Bulgaria: Rhodope Mts [ZSM]. England: Leicester; near London, Dorking [BMNH]. Georgia: $20 \mathrm{~km}$ NE Adigeni, $1800 \mathrm{~m}$; Borjomi; SW Akhalts'ikhe, Vale [SIZK]. Germany [ZSM]: Lower Saxony (Hedemünden), Bavaria (Andechs; Walchensee). Italy [ZSM]: Trentino-Alto Adige (W Rowereto, Riva del Garda, $80 \mathrm{~m}$ ), Veneto reg. (Verona distr., Garda, 300-450 m). Latvia: Aizpute; Bauska. Lithuania [IEV]: Vilnius; Jurbarko. Russia: Smolensk reg. (Smolenskoe poozer'e National Park), Stavropol' reg. (20 km E Stavropol'; Shpakovskoe), Stavropol' reg. (Teberdinskiy Nature Reserve), Saratov reg. (Krasnyy Kut, Dyakovka). Slovenia: Bohinjska Bela [BMNH]. Spain: Valencia (Jijona, $980 \mathrm{~m}$ [CEUA]). Ukraine: Sumy reg. (Mikhailovskaya Tselina nature reserve), Kherson reg. (Chernomors'kij nature reserve [SIZK]), Crimea (Crimean nature reserve [SIZK]).

Distribution. Europe, Caucasus, Turkey. 
Biology. Host unknown. Flight period from late April to late July.

\section{P. monticola Szépligeti, 1899}

Phradis monticola Szépligeti, 1899. Lectotype.+

Material. 57 우 and $\delta$ examined. Bulgaria: Rhodopi [ZSM]. Germany: Hessen (Frankfurt [ZSM]). Italy [ZSM]: TrentinoAlto Adige ("St. Peter/Ahrntal", 1275-1380 m; Partschins, 1000 m). Lithuania [IEV]. Poland: Pieniny [SWC]. Russia: KarachayCherkessia (Arkhyz), Alania (Tsey, 2200 m). Slovakia: Bratislava, Devinská Kobyla nature reserve. Ukraine: Ternopil' reg. (Medobory nature reserve [SIZK]), Crimea (SE Simferopol: Pereval'noye; Chatyr-Dag Mts; General'skoye; Crimean nature reserve [SIZK]).

Distribution. Europe and Russian Caucasus.

Biology. Host unknown. Flight period from May to late August.

\section{P. morionellus (Holmgren, 1860)}

Thersilochus morionellus Holmgren, 1860. Lectotype ㅇ.

Isurgus lanceolatus Szépligeti, 1899. Syntypes đo. Lectotype not designated.

Isurgus oudemansi Smits van Burgst, 1913. Holotype 오 .

Material. $216 \%$ and $\delta$ examined. Austria: Vienna. Belarus: NE Brest, Pruzhany [ZMUM]; "Priluki". Bulgaria [ZSM]: Rhodopi; SE Plovdiv, Katunitsa. England: N Cambridge, Manea, on Brassica juncea infested with Meligethes aeneus \& Ceuthorhynchus sp.; near Cambridge, Trumpington, on Brassica juncea infested with Meligethes aeneus \& Ceuthorhynchus sp. [BMNH]. Germany [ZSM]: Hessen (Witzenhausen), Lower Saxony (Hedemünden; Lippoldshausen), Baden-Württemberg (Emmendingen, 310-360 m), Bavaria (Munich, Schloss Nymphenburg; Oderberg). Kazakhstan: Staropyatigorskoe, Tarbagatay Mts. Latvia: Bauska. Lithuania [IEV]: "Kowno [Kaunas] Georgenburg"; Vilnius; Trakai; SE Rokiškis, Bagdoniškis; Piktupenai; Joniškis; Pasvalys; Žagarè. Moldova: Kishinev [SIZK]. Poland [SWC]: Trzcianne; "Batalionowa Łaka at Białystok". Russia: Kaliningrad reg. (Courish spit, Rybachiy), Yaroslavl' reg. (25 km S Yaroslavl'), Bryansk, Arkhangel'sk reg. (Maloshuyka), Moscow reg. (Kropotovo [ZMUM]; Zibrovo [ZMUM]), Kursk, Smolensk reg. (Smolenskoe poozer'e National park, Przheval'skoe), Stavropol' reg. (Shpakovskoe), Chechnya (Itum-Kali), Alania (Nuzal, Ardon river; Vladikavkaz [SIZK]), Krasnodar reg. (Caucasus Nature Reserve, $15 \mathrm{~km} \mathrm{~W}$ Guzeripl'), Khabarovsk reg., Primorskiy reg. Sweden: Lectotype [MZLU]; Upland, Uppsala [ZSM]. Turkmenistan: Gyzylarbat [Kizil-Arvat]. Ukraine: Zakarpatska reg. [SIZK], Vinnytsya reg. [SIZK], Poltava reg., Kherson reg. [SIZK], Crimea, Kharkiv reg. [SIZK].

Distribution. Common Transpalaearctic species. Tunisia, almost whole of Europe, Turkey, Caucasus, Kazakhstan, Central Asia (Turkmenistan), Russian southern Siberia and south of Far East.

Biology. Parasitoid of Meligethes aeneus F., M. difficilis Heer, M. viridescens F., M. symphyti Heer, M. coracinus Sturm and M. nigrescens Stephens (=picipes Sturm) (Nilsson \& Anderson, 1987; Osborne, 1960; Šedivý, 1983; Williams et al., 1984). Flight period in Europe from early May to August.

\section{P. nigritulus (Gravenhorst, 1829)}

Porizon nigritulus Gravenhorst, 1829. Lectotype + . Ischnobatis albipennis Szépligeti, 1899. Lectotype ô.
Material. $78 q$ and $\delta$ examined. Bulgaria [ZSM]: Rhodope Mts (Rila Monastery, $1150 \mathrm{~m}$; "Trajanovi vrata"). Germany: Bavaria (Gungolding [ZSM]). Italy [ZSM]: Trentino-Alto Adige (Merano [Meran], $700 \mathrm{~m}$ ), Sicilia Is. (Etna, south slope). Kazakhstan: West-Kazakhstan reg. (Har'kin); Astana reg. (Zharkol' and Tengiz lakes; Kökshetaū [Kokchetau] Mts; Borovoye). Mongolia: $30 \mathrm{~km}$ E Ulaanbaatar [SC]. Russia: Kaliningrad reg. (Baltiysk [IEV]), St. Petersburg, Karelia, Voronezh reg. (Voronezh; Voronezh nature reserve [ZMUM]), Sverdlovsk reg. (Nizhniy Tagil; Baranchikskiy), Altay reg. (Kosh-Agach), Krasnoyarsk, Chita reg., Primorskiy reg., Sakhalin Is. [SIZK]. Ukraine: Donetsk reg. (Prishib).

Distribution. Transpalaearctic species. Europe, Turkey, Kazakhstan, Russian Siberia and south of Far East, Mongolia.

Biology. Host unknown. Flight period in Europe from May to August.

\section{P. obscuripes Horstmann \& Kolarov, 1988}

Phradis obscuripes Horstmann \& Kolarov, 1988. Holotype 9.

Distribution. Bulgaria.

Biology. Host unknown. Flight in July.

P. pesenkoi Khalaim, 2007

Phradis pesenkoi Khalaim, 2007. Holotype + .

Material. Ukraine: Crimea, Karagach Mts ( 79 , holotype and paratypes).

Distribution. Ukraine (Crimea).

Biology. Host unknown. Flight in May and June.

\section{P. polonicus Horstmann, 1981}

Phradis polonicus Horstmann, 1981. Holotype + .

Material. $31 q$ and $\delta$ examined. Germany: Lower Saxony (Meensen [ZSM]). Lithuania [IEV]: Vilnius; Jeruzalès. Moldova: Panasheshty, Kodru nature reserve [SIZK]. Poland: Pieniny and Krościenko, $3 \uparrow$ and 20 (paratypes) [HC, SWC]. Russia: Leningrad reg. (55 km S St. Petersburg, Krasnitsy), Novgorod reg. (20 km NW Pestovo, Tychkino), Moscow reg., Khabarovsk reg., Primorskiy reg. Ukraine: Luhans'k reg. (Antratsit; Proval'skaya steppe nature reserve, $15 \mathrm{~km}$ E Sverdlovs'k [SIZK]; Kondrashevka [SIZK]).

Distribution. Transpalaearctic species. Europe, south of Russian Far East.

Biology. Host unknown. Flight period in Europe from April to early June.

\section{P. punctipleuris Horstmann, 1971}

Phradis punctipleuris Horstmann, 1971. Holotype.+

Material. $36 \%$ and $4 \sigma^{\star}$ examined. Armenia: Yerevan [Erevan]. Bulgaria: Rhodopi [ZSM]. Georgia: 20 km NE Akhmeta, $1800 \mathrm{~m} ; 20 \mathrm{~km}$ NE Adigeni; Mokhe; Tsagveri; $10 \mathrm{~km} \mathrm{~W}$ Akhalts'ikhe, Vale [SIZK]. Germany: Hessen (Frankfurt [ZSM]). Italy: Trentino-Alto Adige (W Rowereto, Riva del Garda, 250-500 m, 19 and 20, paratypes [ZSM]). Hungaria: "Marmaros". Kazakhstan: Astana reg. (Kurgal'dzhin). Kyrgyzstan: $20 \mathrm{~km}$ E Talas. Russia: Alania (Tsey, $2200 \mathrm{~m}$ ), Bashkortostan (Bashkiriya nature reserve [SIZK]). Slovakia: S Ružomberok, Biely Potok [BMNH]. Slovenia: Podčetrtek [ZSM]. Ukraine: Ternopil' reg. (Medobory nature reserve), Crimea (Crimean nature reserve [SIZK]), Kharkiv reg. (Krasnokut distr. [SIZK]), Donetsk reg. (Krivaya Luka [SIZK]).

Distribution. Europe, Caucasus, Kazakhstan, Central Asia (Kyrgyzstan). 
Biology. Host unknown. Flight period in Europe from end of April to August.

\section{P. punctus Khalaim, 2007}

Phradis punctus Khalaim, 2007. Holotype.+

Material. Azerbaijan: Ordubad, Akdara, 2000 m (1 , holotype).

Distribution. Caucasus (Azerbaijan).

Biology. Host unknown. Flight in May.

\section{P. rufiventris Horstmann, 1981}

Phradis rufiventris Horstmann, 1981. Holotype $q$.

Material. 22 examined. Kazakhstan: S Almaty, Almaarasan; Tarbagatay Mts, Staropyatigorskoe. Lithuania: Zuninto nature reserve [IEV]. Moldova: Kishinev. Poland [SWC]: Czarna Białostocka, "Jesionowe Góry"; Kulikówka. Russia: Kemerovo reg. (Prokop'yevsk), Irkutsk reg. Ukraine: Luhans'k reg. (Derkul river, Nizhnyaya Il'enka [SIZK]; 15 km E Sverdlovs'k, Proval'skaya steppe nature reserve; Antratsit).

Distribution. Europe, Kazakhstan, Russian southern Siberia.

Biology. Host unknown. Flight period from early May to late June.

\section{P. terebrator Horstmann, 1981}

Phradis terebrator Horstmann, 1981. Holotype $q$.

Material. 11 q examined. Bulgaria: NE Asenovgrad, Gornoslav [HC, ZSM]. Kazakhstan: Quaraghandy reg. Kyrgyzstan: Aryslanbob [Arslanbob]. Lithuania: "Biszis" [IEV]. Russia: Bashkortostan (Bashkiriya nature reserve [SIZK]). Ukraine [SIZK]: Ternopil' reg. (Medobory nature reserve), Crimea (Crimean nature reserve, Svetlaya Polyana).

Distribution. Europe, Kazakhstan and Central Asia (Kyrgyzstan).

Biology. Host unknown. Flight period from end of May to July.

\section{P. thyridialis Horstmann, 1981}

Phradis thyridialis Horstmann, 1981. Holotype 오.

Material. $7 q$ and $6 \hat{0}$ examined. Georgia: $20 \mathrm{~km} \mathrm{NE}$ Adigeni, 1800 m. Hungary: Diósjenő, 19 , paratype [HC]. Italy: Lombardy (Pizzighettone), Veneto (N Vicenza, Piovene, 900-1300 m [ZSM]). Kazakhstan: Kökshetaū [Kokchetav] reg. (Vorob'yevka [SIZK]). Russia: Karelia, Moscow reg. (Mamontovka), Chelyabinsk reg. (Il'menskiy nature reserve).

Distribution. Europe, Caucasus, Urals, North Kazakhstan.

Biology. Host unknown. Flight period from May to early July.

\section{P. toreador Aubert, 1986}

Phradis toreador Aubert, 1986. Holotype + .

(Figs 32-39)

Material. France: Vendée (Longeville, $19[\mathrm{BMNH}])$. Spain: Valencia (Alberique, 19 , holotype [MZLS]).

Distribution. Western Europe (Spain, France, Italy).

Biology. Host unknown. Flight in August and September; type specimen was collected in Spain (Valencia) at the end of February.

\section{$P$. vinosus Khalaim, 2007}

Phradis vinosus Khalaim, 2007. Holotype.+

Material. Georgia: $20 \mathrm{~km}$ NE Adigeni (1 + , holotype).
Distribution. Caucasus (Georgia).

Biology. Host unknown. Flight period in June.

\section{DISCUSSION}

In this paper a new illustrated key to 24 European species is provided, which is thirteen species more than were included in the last key to the European species of Phradis published by Horstmann (1971). The aim of this contribution is to provide a complementary key to the Russian Far East species key published by Khalaim (2007a) so that all Palaearctic species can be identified.

The new species $P$. mediterraneus is similar to $P$. monticola and $P$. corsicator, but differs in having a propodeum with the basal area transverse, about twice as wide as long, and the ovipositor sheath about as long as first tergite, but of special interest is the series of apical external finger like structures on the fourth to sixth flagellomeres in both sexes (Figs 23, 29). These structures are also found in $P$. toreador (Fig. 33) and they are probably present in many other Tersilochinae (e.g., Barycnemis sp., S. Bordera, unpublished). Similar hook-like small structures on the subbasal flagellomeres have been recorded in species of the subfamily Phrudinae (Vikberg \& Koponen, 2000). The finger like structures on the flagellum of Phradis could be sensory structures, but their function is unknown and further studies are needed to demonstrate whether they are common in Tersilochinae and their function, both in males and females.

Fourteen species of Phradis that occur in the Far East of Russia, Siberia, Mongolia, Kazakhstan and Kyrgyzstan have not been recorded from Europe (Khalaim, 2007a). Of the twenty four species recorded from Europe, seven have a transpalaearctic distribution (29\%), nine occur in Europe including Caucasus (38\%), two species are known from Caucasus only (8\%), and six occur in Europe but their areas of distribution reach Kazakhstan, Kyrgyzstan, Tadzhikistan, or southern Siberia (25\%). Generally the European fauna of Phradis is well studied so these ratios are more or less an accurate reflection of the real distribution of the European species.

Five species, $P$. brevicornis, $P$. brevis, $P$. interstitialis, $P$. minutus and $P$. morionellus, are widely distributed in Europe (probably Pan-European), and among them two species, $P$. brevis and $P$. morionellus, are the most abundant. The following species are rare or have a rather restricted distribution: $P$. mediterraneus sp. n. (Spain), $P$. corsicator and P. toreador (Spain and France), P. obscuripes (Bulgaria), $P$. pesenkoi (Ukraine), $P$. punctus and $P$. vinosus (Caucasus), and $P$. decameron (Ukraine and Caucasus).

Adults of almost all Phradis species are most active from April to July, but may be collected until autumn, except $P$. toreador which has a flight period in August, September and February.

ACKNOWLEDGEMENTS. We are grateful to the curators of the Musée Cantonal de Zoologie (Lausanne, Switzerland), Museum of Zoology of Lund University (Sweden), the Natural History Museum (London, UK), Zoologischen Staatsammlung München (Germany), Museo Nacional de Ciencias Naturales 
(Madrid, Spain), Institute of Zoology (Kiev, Ukraine), and Zoological Museum of Moscow State University (Moscow, Russia) for the loan of Phradis type and non-type material from the collections in their care. We are also thankful to K. Horstmann (Würzburg, Germany), K. Schöenitser (München, Germany), M. Schwarz (Kierchlag, Austria), A. Lozan (České Budějovice, Czech Republic), J. Sawoniewicz (Białystok, Poland), V. Jonaitis (Vilnius, Lithuania), A.G. Kotenko and N.B. Narolskiy (Kiev, Ukraine), and A.E. Humala (Petrozavodsk, Russia) for the loan of valuable material. We want to express our sincere thanks to the staff of the Cabañeros National Park, Sierra Mariola Natural Park and Font Roja Natural Park (Spain) for providing facilities and granting permission for us to collect material in these protected areas. This research was supported partially by Project GV06/271 from Conselleria d'Empresa, Universitat i Ciència of Generalitat Valenciana, Project 040/2002 from Ministerio de Medio Ambiente (Spain), the Russian Foundation for Basic Research (no. 07-04-00454) and the Presidium of the Russian Academy of Sciences Program "Origin and evolution of Biosphere, Subprogram II".

\section{REFERENCES}

AuberT J.F. 1969: Deuxième travail sur les Ichneumonides de Corse (Hymenoptera). Veröff. Zool. Staatssamml. (München) 13: $27-70$.

AUBERT J.F. 1986: Quatorze Ichneumonides pétiolées inédites ou synonymes. Bull. Soc. Entomol. Mulhouse (avril-juin): $17-25$.

Bridgman J.B. 1889: Further additions to the Rev. T.A. Marshall's catalogue of British Ichneumonidae. Trans. Entomol. Soc. London 1889: 409-439.

BrischKe C.G.A. 1880: Die Ichneumoniden der Provinzen Westund Ost-Preussen. Schrift. Naturf. Ges. Danzig 4: 108-210.

Снао H.F. 1976: An Outline of the Classification of the Ichneumon-flies of China (Hymenoptera: Ichneumonidae). Scientific Publisher, Beijing, 413 pp. [in Chinese].

EADY R.D. 1968: Some illustrations of microsculpture in the Hymenoptera. Proc. R. Entomol. Soc. Lond. 43(4-6): 66-72.

FöRSTER A. 1869: Synopsis der Familien und Gattungen der Ichneumoniden. Verh. Naturhist. Ver. Preussischen Rheinlande Westfalens 25[1868]: 135-221.

GAULd I.D. 1984: An Introduction to the Ichneumonidae of Australia. British Museum (Natural History), London, 413 pp.

Gauld I \& Bolton B. 1988: The Hymenoptera. British Museum (Natural History), Oxford University Press, Oxford, $332 \mathrm{pp}$.

Gravenhorst J.L.C. 1829: Ichneumonologia Europaea. Pars III. Vratislaviae, $1097 \mathrm{pp}$.

Hellén W. 1958: Die Tersilochinen Finnlands (Hym., Ichn.). Notul. Entomol. 38: 4-23.

Holmgren A.E. 1860: Försök till uppställning och beskrifning af de i Sverige funna Ophionider. (Monographia Ophionidum Sueciae). Kongliga Svensk. Vet. Acad. Handl. 2(8): 1-158.

Horstmann K. 1971: Revision der europäischen Tersilochinen I (Hymenoptera: Ichneumonidae). Veröff. Zool. Staatssamml. (München) 15: 45-138.
Horstmann K. 1981: Revision der europäischen Tersilochinen II (Hymenoptera: Ichneumonidae). Spixiana (Suppl. 4), 76 pp.

Horstmann K. \& Kolarov J. 1988: Neue Tersilochinen-Arten aus Bulgarien (Hymenoptera: Ichneumonidae). Spixiana 10: 271-277.

Khalaim A.I. 2002: A new species of the genus Phradis Förster, 1869 from the USA (Hymenoptera: Ichneumonidae: Tersilochinae). Russ. Entomol. J. 11: 221-222.

Khalaim A.I. 2004: A new species of the genus Phradis Förster with 10-11 segmented antenna from the Ukraine (Hymenoptera: Ichneumonidae, Tersilochinae). Zoosyst. Ross. 12: 243-244.

Khalaim A.I. 2007a: 17. Subfamily Tersilochinae. In Lelej A.S. (ed.): Key to the Insects of Russian Far East. Vol. IV. Neuropteroidea, Mecoptera, Hymenoptera. Pt 5. Dal'nauka, Vladivostok, pp. 566-597 [in Russian].

Khalaim A.I. 2007b: First records of Meggoleus, Heterocola and Phradis (Hymenoptera: Ichneumonidae: Tersilochinae) from the Afrotropical region, with description of four new species. Afr. Invertebr. 48: 101-110.

Nitsson C. \& ANDREAsson B. 1987: Parasitoids and predators attacking pollen beetles (Meligethes aeneus F.) in spring and winter rape in southern Sweden. IOBC/WPRS Bull. 10: 64-73.

OsBorne F. 1960: Observations on the natural enemies of Meligethes aeneus (F.) and M. viridescens (F.) [Coleoptera: Nitidulidae]. Parasitology 50: 91-110.

ŠEDIVÝ J. 1983: Tersilochinae as parasitoids of insect pests of winter rape (Hymenoptera: Ichneumonidae). Contrib. Am. Entomol. Inst. 20: 266-276.

Smits van Burgst C.A.L. 1913: Ichneumonidae II. Entomol. Ber. 3: 363-367.

Strobl G. 1904: Ichneumoniden Steiermarks (und der Nachbarländer). V. Fam. Ophionidae. Mitt. Naturw. Ver. Steiermark (Graz) 40[1903]: 43-160.

SzÉPLIGETI G. 1899: Beiträge zur Kenntniss der ungarischen Ichneumoniden. Term. Füzetek 22: 213-246.

THOMson C.G. 1889: XL. Försök till gruppering och beskrifning af arterna inom slägtet Porizon (Grav.). Opusc. Entomol. (Lund) 13: 1354-1400.

Townes H.K. 1969: The genera of Ichneumonidae, Part 1. Mem. Am. Entomol. Inst. No. 11, 300 pp.

Townes H.K. 1971: The genera of Ichneumonidae, Part 4. Mem. Am. Entomol. Inst. No. 17, 372 pp.

ViKBerg V. \& Koponen M. 2000: On the taxonomy of Seleucus Holmgren and the European species of Phrudinae (Hymenoptera: Ichneumonidae). Entomol. Fenn. 11: 195-228.

Williams R.N., Weiss M.J., Kehat M. \& Blumberg D. 1984: The hymenopterous parasites of Nitidulidae. Phytoparasitica 12: 53-64.

Yu D.S., van Achterberg K. \& Horstmann K. 2005: World Ichneumonoidea 2004. Taxonomy, Biology, Morphology and Distribution. CD/DVD. Taxapad, Vancouver, Canada. www.taxapad.com

Received April 29, 2008; revised and accepted October 1, 2008 